\title{
Circuit Area Optimization in Energy Temporal Sparse Scenarios for Multiple Harvester Powered Systems
}

\author{
Raul G. Cid-Fuentes*, Albert Cabellos-Aparicio ${ }^{\dagger}$ and Eduard Alarcón* \\ * Departament d'Enginyeria Electrònica, ${ }^{\dagger}$ Departament d'Arquitectura de Computadors \\ NaNoNetworking Center in Catalunya (N3Cat) \\ Universitat Politècnica de Catalunya, 08034 Barcelona, Spain \\ Email: \{rgomez, acabello \} @ac.upc.edu, eduard.alarcon@upc.edu
}

\begin{abstract}
Multi-source energy harvesters are gaining interest as a robust alternative to power wireless sensors, since the sensor node can maintain its operation regardless of the fact that one of its energy sources might be temporarily unavailable. Interestingly, and less explored, when the energy availability of the energy sources present large temporal variations, combining multiple energy sources reduce the overall sparsity. As a result, the performance of a multiple energy harvester powered sensor node is significantly better compared to a single energy source which harvests the same amount of energy. In this context, a circuit area optimization framework for multiple source energy harvesting powered systems is proposed. This framework takes advantage of this improvement in performance to provide the optimal amount of energy harvesters, the requirements of each energy harvester and the required energy buffer capacity, such that the overall area or volume is minimized. As the results show, by conducting a joint design of the energy harvesters and the energy buffer, the overall area or volume of a sensor node can be significantly reduced.
\end{abstract}

\section{INTRODUCTION}

Energy harvesting is a technology which is considered as a firm candidate to enable perpetual wireless sensor networks [1]. Recently, multi-source energy harvesters are gaining interest as a robust alternative to power wireless sensors [2], [3], [4]. As an example, authors in [2] provide a platform for vibration and solar energy harvesting. These platforms are more robust than the single-source ones. Indeed, if a certain energy source renders unavailable for a certain time period, due to the time asynchronicity among energy sources the sensor node can still maintain its normal operation.

One of the main challenges in the design of energy harvesting enabled sensor nodes lies in the dimensioning of both the energy harvesting and energy buffer units. Considering both subsystem units to be sufficiently large solves undesired interruptions during the normal operation of the sensor node and, accordingly, on the wireless network.

Unfortunately, over-dimensioning precludes desirable miniaturization of the sensor nodes, caused by the relatively small power densities of existing ambient energy sources and low energy density of energy buffers [5], [1]. As an example, in order to harvest $0.2 \mathrm{~mW}$ vibrational energy and to store 1 $\mathbf{J}$ of energy, an energy harvester of approximated $1 \mathrm{~cm}^{2}$ and an energy buffer of approximated $2 \mathrm{~cm}^{3}$ would be required.

An additional, but less explored, advantage of heterogeneous multiple-source energy harvesters, which aids the miniaturization of the sensor nodes, is that when the ambient energy

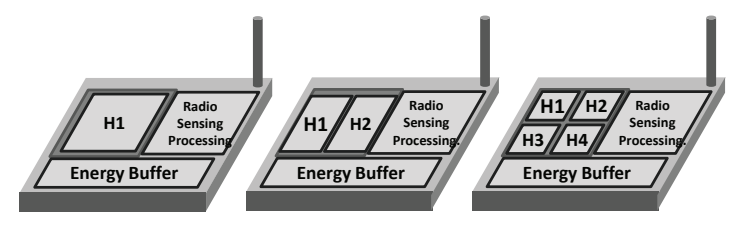

Fig. 1. Multi-source energy harvesting enabled wireless sensors.

presents a large temporal variations (i.e., the harvested power randomly varies over a wide range along time) the combination of multiple statistically independent energy sources lowers the sparsity of the overall energy which is harvested. This causes that sensor nodes which are powered by multisource energy harvesters outperform in contrast to singlesource configurations. Equivalently, the requirements in terms of energy buffer capacity can be relaxed, while maintaining the same performance. As an example, Fig. 1 shows three sensor nodes that implement one, two or four energy harvesters which occupy the same overall area, in a chip-like planar implementation

In this paper we present an analytic framework which optimizes the overall occupied area by the energy harvesting and energy buffer units. In particular, this model accounts for the requirements and capabilities of the sensor units, and provides (i) the optimal number of energy harvesters, (ii) their size and (iii) the energy buffer capacity, such that the overall area of the sensor node is minimized, while still meeting the user-defined requirements of the communications unit.

As a result, in addition to the aforementioned benefits in terms of robustness when using multiple energy harvesters, this framework shows that using multi-source energy harvesters in energetically sparse scenarios results in a significant reduction of the sensor size.

The structure of this paper is organized as follows: In Sec. II we present the sparse energy sources. In Sec. III we compare the performance of single-source to multi-source energy harvesters powered sensor nodes. In Sec. IV we present the circuit-area model to be optimized, while in Sec. V we evaluate this model in a particular case. Finally, in Sec. VI we conclude our work.

\section{SPARSE ENERGy SOURCES}

Ambient energy is generally generated by the aggregation of an extensive number of physical entities which simultaneously 


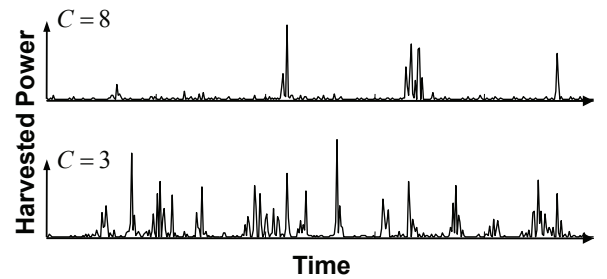

Fig. 2. Harvested power from a sparse ambient source of crest factor of (upper) $C=8$ and (lower) $C=3$.

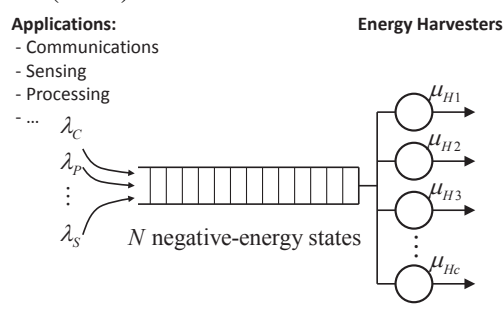

Fig. 3. The negative energy queue model.

radiate power [1]. Then, the random contribution of each entity, in both magnitude and time duration, entails a timevarying character in the aggregated power.

Accordingly, we refer as a sparse energy source to any physical phenomena which produces an aggregated power in a sparse, time-varying manner, such that this power cannot be known or estimated and the magnitude of the instantaneous power falls within a wide range. In fact, sparse energy sources are present in a wide variety of physical phenomena. Among others, acoustic energy, mechanical, vibrational or RF energy [6], [7], [8] are considered representative examples of such sources, when considering a large time scale.

In this work, we propose the power crest factor as a metric to enable the comparison of performance among ambient energy sources. The power crest factor models the relation between the average peak power over the average power. We define the power crest factor as:

$$
C=\frac{\overline{P_{\text {peak }}}}{P_{H}},
$$

where $\overline{P_{\text {peak }}}$ is the average peak power and $P_{H}$ refers to the average harvested power. Fig. 2 shows examples of two random energy sources with different crest factor $(C=8$ and $C=3$ ). As it is shown, a large crest factor provides short but powerful bursts of energy, while leaving large inter-burst times where the available energy is far below the average value.

\section{Multiple Source Energy Harvesters}

Combining independent energy sources, the sparsity of the overall process is reduced and thus the energy fadings are potentially reduced, as well. In this section we evaluate the improvement on performance that using multiple energy harvesting platforms has when contrasted to single harvester platforms.

\section{A. Energy Model}

In order to evaluate this performance, we use the negative energy queue model which is shown in Fig. 3. This Markovbased model is similar to other existing energy models for energy harvesting [9], [10], [11], [12]. However, as it is shown, the arrivals of this queue are generated by the set of applications of the sensor node. i.e., every time an application spends one unit of energy, it generates an arrival of negative energy. Each kind of application has an associated generation rate in power units (e.g. $\lambda_{C}$ for communications, $\lambda_{P}$ for processing and $\lambda_{S}$ for sensing). On the other hand, each harvester has an associated service time, $T_{H}=E_{s} / \mu_{H}$, which is the time that this energy harvesting unit needs to process one negative energy packet, where $E_{s}$ is the energy of a negative energy packet and $\mu_{H}$ the energy harvesting rate in power units.

Thus, the number $N$ of negative energy states is related to the energy buffer capacity, $C_{B}$ as:

$$
N=\frac{C_{B}}{E_{s}}
$$

Additionally, if at a certain time $t_{k}$ the queue has $L^{k}$ negative energy packets, then the energy state $s^{k}$ at the energy buffer is given by:

$$
s^{k}=C_{B}-L^{k} E_{s} .
$$

\section{B. Evaluation Metrics}

As the main metrics that we have used to evaluate the performance of the sensor node, we first have defined the energy utilization, $\rho_{e}$ in Energy-Erlangs (E2), which relates the amount of power which is required, $P_{C}$, in contrast to the harvested power, $P_{H}$, as:

$$
\rho_{e}=\frac{P_{C}}{P_{H}} .
$$

Secondly, we define the energy outage probability, $p_{\text {out }}$, as the probability of depleting the energy buffer, and thus temporarily interrupting the communication.

\section{Performance of a Multiple Source Energy Harvester}

In order to provide realistic results to justify the importance of such platforms, we have considered an average communications rate of $\lambda_{c}=P_{C}=100 \mu \mathrm{W}$. Then, we have considered each negative energy packet to be of $10 \mu \mathrm{J}$. Finally, we have set the overall harvesting rate $N \mu_{H}=P_{H}=P_{C} / \rho$, where $\rho_{e}$ has been set as an evaluation parameter. Therefore, each harvester harvests an average power of $P_{C} / \rho_{e} N$. These energy harvesting rates can be achieved by means of vibrational harvesters [1].

In order to generate the sparse energy sources, we have approximated the ambient energy by a random process generated by exponentially distributed energy bursts of power $P_{H} C / N$, with an inter-burst time of $0.1 / C$ seconds. An exponentially distributed random process has been chosen as it presents the largest entropy, thus estimating the worst case [6].

Fig. 4 and Fig. 5 compare the improvement over $p_{\text {out }}$ that using multiple harvesters has as a function of the energy buffer capacity, $C_{B}$, for a crest factor of $C=10$ and $C=100$ respectively. These results have been obtained by assuming in the negative energy queue model $\rho_{e}=0.9$. As it is shown, there is a clear improvement, since varying from one to five harvesters, the energy buffer capacity can be reduced from 


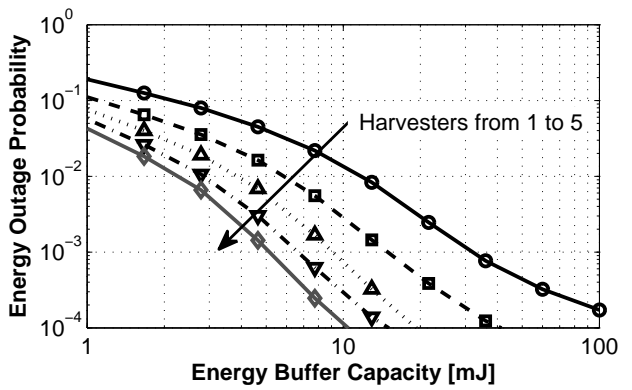

Fig. 4. Energy Outage Probability as a function of the energy buffer capacity. $\rho_{E}=0.9 \mathrm{E} 2$ and $C=10$.

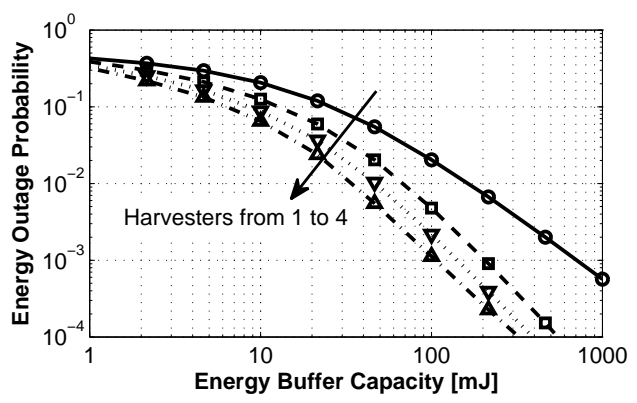

Fig. 5. Energy Outage Probability as a function of the energy buffer capacity. $\rho_{E}=0.9 \mathrm{E} 2$ and $C=100$.

$30 \mathrm{~mJ}$ to just $5 \mathrm{~mJ}$ and from $600 \mathrm{~mJ}$ to just $100 \mathrm{~mJ}$, while still maintaining $p_{\text {out }}<10^{-3}$.

In addition to this, Fig. 7 and Fig. ?? compare this improvement as a function of the $\rho_{e}$ for crest factors of $C=10$ and $C=100$ respectively. In order to obtain these results, the energy buffer capacity has been set to $C_{B}=10 \mathrm{~mJ}$ in Fig. 7 and to $C_{B}=100 \mathrm{~mJ}$ in Fig. ??. As it is shown, multi-source energy harvesters are able to provide similar performance, but at larger $\rho_{e}$ values and, therefore, requiring smaller energy harvesting area.

As a result, we observe that multi-source energy harvesters can help reducing both the energy buffer capacity, as well as the energy harvesting requirements, while still providing the required performance.

\section{Circuit Area Model}

As seen in the previous section, additional energy harvesters have a positive impact upon the performance. Nonetheless, this technique produces a non-negligible area overhead, since

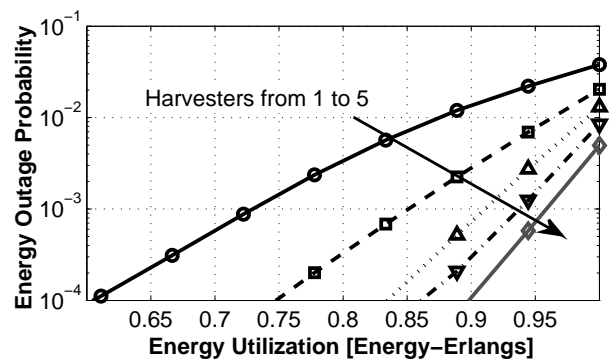

Fig. 6. Energy Outage Probability as a function of the energy utilization. $C_{B}=10 \mathrm{~mJ}$ and $C=10$.

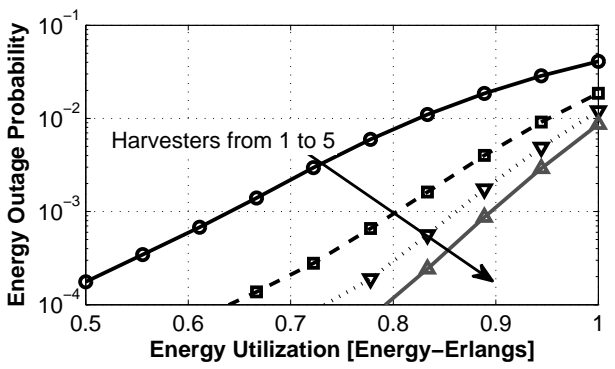

Fig. 7. Energy Outage Probability as a function of the energy utilization. $C_{B}=100 \mathrm{~mJ}$ and $C=100$.

TABLE I

VALUES USED IN THE OPTIMIZATION FRAMEWORK

\begin{tabular}{l|c|c}
\hline Parameter & Value & units \\
\hline$A_{H 0}$ & 0.02 & $\mathrm{~cm}^{2}$ \\
$A_{N H}$ & .01 & $\mathrm{~cm}^{2}$ \\
$A_{N P}$ & 6.66 & $\mathrm{~cm}^{2} \mathrm{~mW}^{-1}$ \\
\hline
\end{tabular}

each energy harvester requires some additional circuitry and separation space.

An additional compromise is that low values of $\rho_{e}$ help reducing the energy buffering capacity at the cost of proportionally increasing the energy harvesting requirements.

These compromises motivate a framework for circuit area optimization which considers the user-defined requirements, the area overhead of multiple harvesters and the energy buffer capacity. In order to do so, we first relate the required power, harvesting power, number of harvesters and energy buffer capacity which are able to achieve the required performance in energy outage probability, through the energy model presented in Sec. III. Afterwards, this is translated into circuit area by means of the following model.

We then define the overall area of the system as:

$$
A_{\text {TOTAL }}=A_{H}+A_{B}+A_{A},
$$

where $A_{H}$ refers to the area of the harvesting unit, $A_{B}$ stands for the area of the energy buffer unit and $A_{A}$ is the area of the applications units (i.e., processing, sensing and communications unit). In particular, since $A_{A}$ is fixed and provided by a certain application, $A_{A}$ is not considered in the following circuit area optimization.

\section{A. Area of the Energy Harvesting Unit}

The area of the harvesting unit depends on mainly two factors, the number of energy harvesters and the power that these aim to harvest. In particular, we linearly approximate the area of the energy harvesting unit by:

$$
A_{H}=A_{H 0}+A_{H N} N_{H}+A_{H P} P_{R} / \rho_{e},
$$

where $A_{H 0}$ refers to a constant area, $A_{H N}$ to the partial contribution of $A_{H}$ with respect to the number, $N_{H}$, of energy harvesters and $A_{H P}$ to the partial contribution of $A_{H}$ with respect to the required power $P_{H}$.

In this work, we have chosen the parameters shown in Table I, which correspond to reasonable values as reported in [1]. 


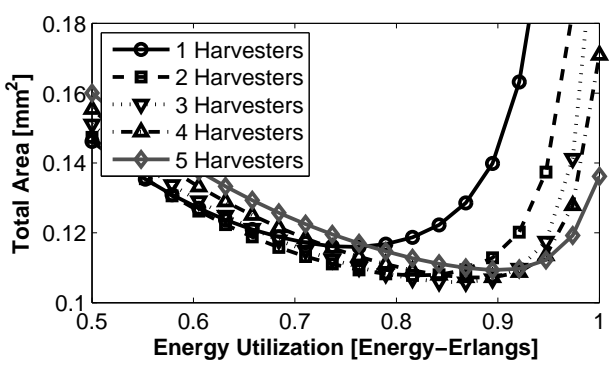

Fig. 8. Overall Area in terms of the Energy Utilization. $C=10$.

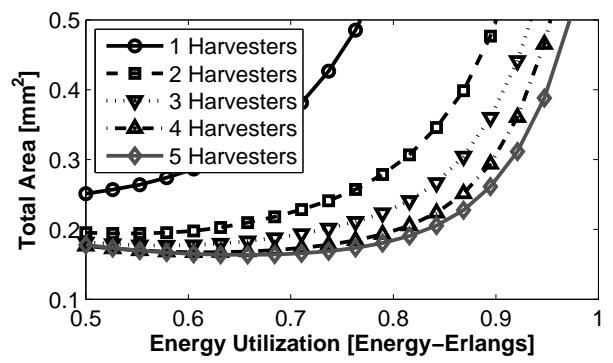

Fig. 9. Overall Area in terms of the Energy Utilization. $C=10$.

\section{B. Area of the Energy Buffer}

In line with recent advancements in energy buffering [5], each technology presents an associated energy density. In this context, we have considered consistent values for this density of $D_{B}=2 \mathrm{~J} / \mathrm{cm}^{-3}$ and a fixed height of $1 \mathrm{~cm}$. Similar to $A_{H}$, we may linearly approximate the overall area of the energy buffer as:

$$
A_{B}=A_{B 0}+C_{B} D_{B},
$$

where $A_{B 0}$ is a fixed area overhead and $C_{B}$ is the required capacity of the energy buffer in $\mathrm{mJ}$ units.

\section{AREa Optimization}

In order to optimize the area, we have simulated the sensor node through the same energy model as described in the previous sections. In addition to this, a target in the energy outage probability has been set to $p_{\text {out }}=10^{-4}$.

Fig. 8 shows the required overall occupied area for the joint energy harvesting and energy buffer unit to meet the user defined requirements in both output power and energy outage probability, when considering a crest factor of $C=10$. As it is shown, the minimum area is found at a $\rho_{E}=0.87 \mathrm{E} 2$, considering three energy harvesters.

Similarly, Fig. 9 shows the results of the circuit-area optimization when considering the same system requirements, but assuming a crest factor of $C=100$. As it is shown, an increase in the crest factor motivates the use of more energy harvesters. In particular, the minimum area is found at a $\rho_{E}=0.66 \mathrm{E} 2$, considering five energy harvesters. The outcome of this design, which are required for the energy harvesting unit and an energy buffer to minimize the area can be found in Table. II for both cases.

\section{CONCLUSION}

Multi-source energy harvesting is gaining popularity as an alternative to power wireless sensor nodes. The benefits that
TABLE II

COMPONENT REQUIREMENTS

\begin{tabular}{l|c|c|c}
\hline Crest Factor & Parameter & Value & Units \\
\hline \hline 10 & Harvesters & 2 & - \\
& Area Harvester (total) & 9.8 & $\mathrm{~mm}^{2}$ \\
& $P_{H}$ (each) & 57.5 & $\mu \mathrm{W}$ \\
& Area Energy Buffer & 2.0 & $\mathrm{~mm}^{2}$ \\
& Capacity Energy Buffer & 10 & $\mathrm{~mJ}$ \\
\hline 100 & Harvesters & 5 & - \\
& Area Harvester (each) & 14.7 & $\mathrm{~mm}^{2}$ \\
& $P_{H}$ (each) & 40 & $\mu \mathrm{W}$ \\
& Area Energy Buffer & 4 & $\mathrm{~mm}^{2}$ \\
& Capacity Energy Buffer & 20 & $\mathrm{~mJ}$ \\
\hline
\end{tabular}

this alternative provides when the ambient energy is largely time-variant is two-fold: on the one hand, it provides robustness to the sensor node, while on the other hand, the sparsity of the overall contribution is reduced, and thus its performance is improved. In this context, circuit area optimization which takes advantage of the improvement in performance of multiplesource energy harvesters has been addressed. As it has been shown, this joint effort can help reducing the overall area, thus enabling a future miniaturization of the wireless sensor nodes.

\section{ACKNOWLEDGEMENTS}

Partial funding from AGAUR FI-DGR grant.

\section{REFERENCES}

[1] S. Sudevalayam and P. Kulkarni, "Energy harvesting sensor nodes: Survey and implications," IEEE Communications Surveys Tutorials, vol. 13 , no. 3, pp. $443-461,2011$.

[2] S. Bandyopadhyay and A. Chandrakasan, "Platform architecture for solar, thermal, and vibration energy combining with mppt and single inductor," IEEE Journal of Solid-State Circuits, vol. 47, no. 9, pp. 2199 -2215, September 2012.

[3] C. Park and P. Chou, "Ambimax: Autonomous energy harvesting platform for multi-supply wireless sensor nodes," in Proc. of the 3rd Annual IEEE Communications Society on Sensor and Ad Hoc Communications and Networks (SECON), vol. 1, 2006, pp. 168-177.

[4] A. S. Weddell, M. Magno, G. V. Merrett, D. Brunelli, B. M. Al-Hashimi, and L. Benini, "A survey of multi-source energy harvesting systems," in Proc. of the Design, Automation Test in Europe Conference Exhibition (DATE), 2013, pp. 905-908.

[5] D. Pech, M. Brunet, H. Durou, P. Huang, V. Mochalin, Y. Gogotsi, P.-L. Taberna, and P. Simon, "Ultrahigh-power micrometre-sized supercapacitors based on onion-like carbon," Nature Nano, vol. 5, no. 9, pp. 651 - 654, September 2010.

[6] M. Win, P. Pinto, and L. Shepp, "A mathematical theory of network interference and its applications," Proceedings of the IEEE, vol. 97, no. 2, pp. $205-230$, feb. 2009.

[7] S. Chalasani and J. Conrad, "A survey of energy harvesting sources for embedded systems," in Proc. of the IEEE Southeastcon, 2008, pp. $442-447$.

[8] A. Hajati, S. Bathurst, H. Lee, and S. Kim, "Design and fabrication of a nonlinear resonator for ultra wide-bandwidth energy harvesting applications," in Proc. of the IEEE International Conference on Micro Electro Mechanical Systems (MEMS), January 2011, pp. 1301 -1304.

[9] R. G. Cid-Fuentes, A. Cabellos, and E. Alarcon, "Energy harvesting enabled wireless sensor networks: Energy model and battery dimensioning," in Proc. of the 7th International Conference on Body Area Networks (BODYNETS), September 2012.

[10] R. Rajesh, V. Sharma, and P. Viswanath, "Information capacity of energy harvesting sensor nodes," in Proc. of the IEEE International Symposium on Information Theory, 31 2011-aug. 5 2011, pp. 2363 -2367.

[11] O. Ozel, K. Tutuncuoglu, J. Yang, S. Ulukus, and A. Yener, "Transmission with energy harvesting nodes in fading wireless channels: Optimal policies," Journal of Selected Areas in Communications, vol. 29, pp. 1732-1743, September 2011.

[12] M. Gorlatova, A. Wallwater, and G. Zussman, "Networking low-power energy harvesting devices: Measurements and algorithms," in Proc. of the IEEE INFOCOM, april 2011, pp. $1602-1610$. 